\title{
FROM RAILWAY RESOURCE PLANNING TO TRAIN OPERATION
}

\author{
a brief survey of complementary formalisations
}

\author{
Martin Penicka* \\ Department of Applied Mathematics, Faculty of Transportation Sciences, \\ Czech Technical University, Na Florenci 25, CZ-11000 Prague \\ penicka@fd.cvut.cz
}

\begin{abstract}
Dines Bjørner*
Department of Computer Science, School of Computing, National University of Singapore, 3 Science Drive 2, Singapore 117543

E-Mail: bjorner@comp.nus.edu.sg, URL: http://www.comp.nus.edu.sg/cs/
\end{abstract}

\begin{abstract}
From seasonal planning via day-to-day train operation to real-time monitoring and control of trains, software applications are becoming increasingly integrated. Timetabling implies train traffic. Train staff rosters and train car maintenance are initially derived from timetables and influences future timetables.

In this extended abstract we shall sketch a formal model of Railway Nets, Timetables, Rosters, Maintenance, Station Interlocking, Line Direction Agreement and Automatic Line Signaling. The last three formal models are based on four integrated formal techniques (RAISE, Petri Nets, Live Sequence Charts and State Charts). The formal sketches are all "backed-up" by either a publication or a research report.
\end{abstract}

Keywords: Railways, Planning, Timetabling, Rostering, Control, Interlocking, Signalling, RAISE, Petri Nets, Live Sequence Charts, State Charts, Technique Integration

\section{Railway System}

A railway system $(\Omega)$ can be modelled as a function from time $(\mathrm{T})$ to states of a railway net $(\mathrm{N})$, to states of all rolling stock (RS), to its timetable (TT), to states of all passengers $(\mathrm{P})$ and freight $(\mathrm{F})$.

\footnotetext{
*This paper was written while the authors were at Inst. of Informatics \& Modelling, Technical University of Denmark, DK-2800 Kgs. Lyngby, Denmark. The first author gratefully acknowledges support from the EU 5FP Network of Excellence Programme: CoLogNET: Computational Logic Network
} 
type

$T, N, R S, T T, P, F$

$\Omega^{\prime}=\mathrm{T} \rightarrow(\mathrm{N} \times \mathrm{RS} \times \mathrm{TT} \times(\mathrm{P} \times \mathrm{F}))$

$\Omega=\left\{\left|r w: \Omega^{\prime} \bullet w f . \Omega(r w)\right|\right\}$

value

wf_ $\Omega: \Omega^{\prime} \rightarrow$ Bool

wf_ $\Omega(\mathrm{rw}) \equiv$ well-formedness of railway states

The well-formedness describes constraints that must be met by any railway system. They are laws of nature, e.g. trains move monotically, net changes states accordingly, etc.

\section{Allocation \& Scheduling of Resources}

\section{From Passenger Statistics to Railway Nets and Timetables}

Passenger statistics (STA) express predicted number of passengers between pairs of geographical centers (C) (urban area were potential passengers live) in time intervals $(\mathrm{T} \times \mathrm{T})$. From a cartographical map (MAP) one can observe the geographical centers and their positions.

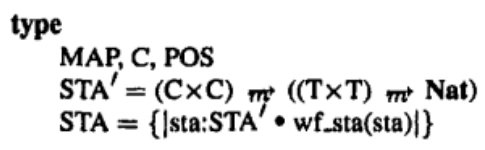

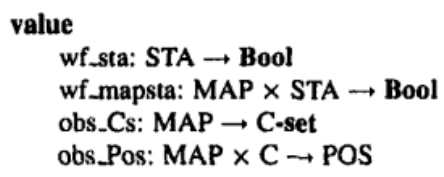

A railway net $(\mathrm{N})$ is composed from stations and lines. Lines $(\mathrm{T})$ connect stations. A railway station $(S)$ is a place where trains stop to allow passengers to enter and get off a train as reflected in timetable.

A timetable (TT) expresses for all planned trains $(\mathrm{Tn})$ their journeys $(\mathrm{J})$. A train journey $(\mathrm{J})$ is a list of line visits (departure time from a station, line, arrival time time to a station, train capacity $(\mathrm{K})$, and possible periodicity (PRD) (e.g. 24hour, or 20 minutes) and restrictions (RST) on the days for which it applies (e.g. Mon-Fri, only or summer season only)).

Now one can define a function genNTT which from a given geographical map and passenger statistics, and according to a given set of predicates $(\mathrm{P})$, generates all possible pairs of nets and timetables (NTT), such that these satisfy the map and the passenger statistics.

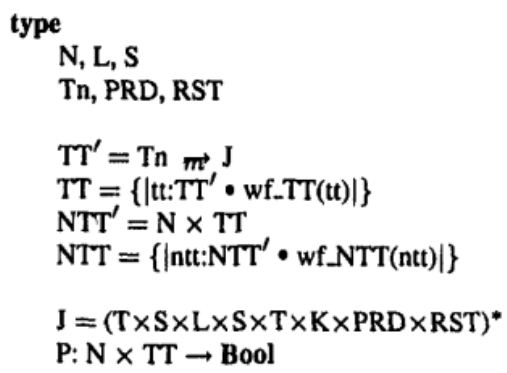

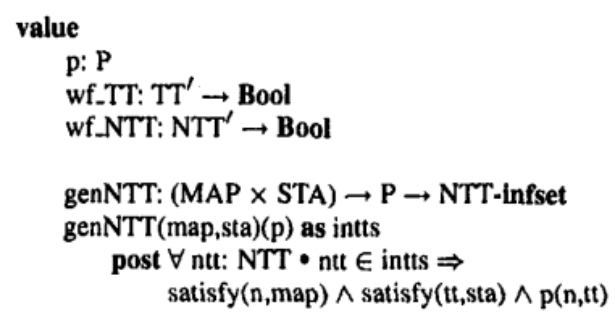




\section{From Nets \& Timetables to Operational Planning}

Let us now define a usually heuristic operational planning process, Planning, which from a given geographical map and passenger statistics according to a given set of predicates (P), generates one possible pair of nets and timetables (NTT).

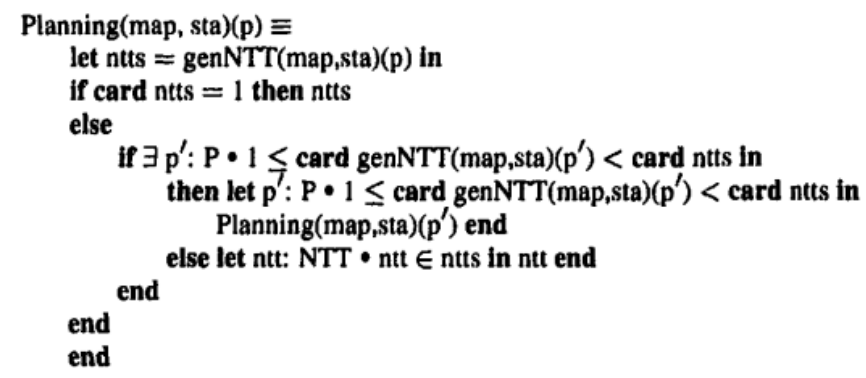

\section{From Timetables and Rolling Stock to Vehicle Scheduling}

Given a net, a timetable and an available rolling stock (RS) one is interested in computing optimal working plans (VWP) for vehicles $(\mathrm{V})$ of rolling stock such that these plans honour the timetable. A set of predicates $(\mathrm{P})$ on rolling stock, nets and timetables has to be satisfied (one can operate electric powered engine only on suitable lines, etc.). We model the set of predicate as one "grand" predicate.
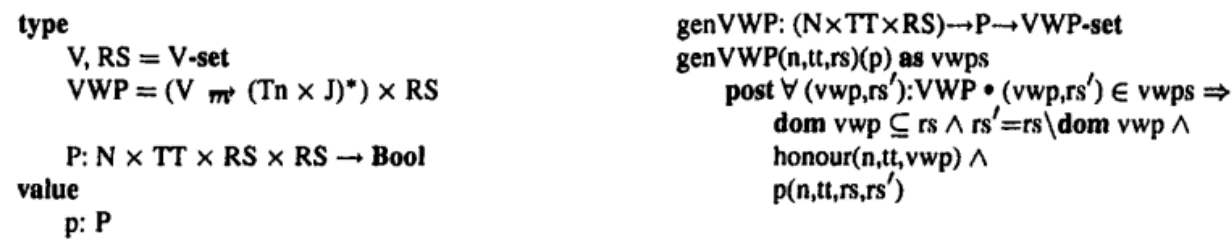

\section{From Timetables and Human Resources to Rostering}

Given a net and a timetable one can determine the number of human resources (HR) of each type (drivers, conductors, etc.) needed to honour the timetable. We refer to [5]. Let us just show, how one can generate a set of working plans (HWP) for railway employees $(\mathrm{H})$. A set of predicates $(\mathrm{P})$ on human resources, nets and timetables has to be satisfied. We model the set of predicate as one "grand" predicate.

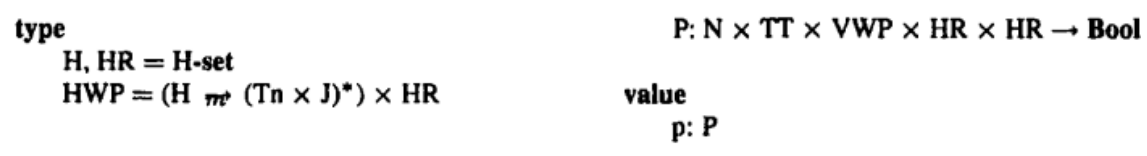


genHWP: $(\mathrm{N} \times \mathrm{TT} \times \mathrm{VWP} \times \mathrm{HR}) \rightarrow \mathrm{P} \rightarrow \mathrm{HWP}-$ set

genHWP(n,tt,vwp,hr)(p) as hwps

post $\forall($ hwp, hr' $): H W P \bullet\left(h w p, h r^{\prime}\right) \in$ hwps $\Rightarrow$ dom hwp $\subseteq$ hr $\wedge$ hr' $=h r \backslash$ dom hwp $\wedge$ honour(n,tt,vwp,hwp,hr) $\wedge$

$\mathrm{p}\left(\mathrm{n}, \mathrm{tt}, \mathrm{vwp}, \mathrm{hr}, \mathrm{hr} \mathrm{f}^{\prime}\right)$

\section{From Timetables to Vehicle Maintenance}

The earlier vehicle working plans did not specify that any vehicle has to undergo preventive maintenance. We now define a set of functions, which modify vehicle working plans to reflect timely maintenance. By a maintenance we understand all regular activities which must be done with rolling stock and according to some rules (R). Each vehicle, according to its type, has associated with it certain types of maintenance tasks to be performed with a frequency which can be expressed by elapsed number of kilometer or operating house since a previous maintenance.

Given a railway net $(\mathrm{N})$, vehicle working plans (VWP), a timetable $(\mathrm{TT})$ and a planning period (TxT) the job is to generate all the possible sets of changes (CS), necessary and sufficient to secure maintenance. Given these sets, one is selected and used for update of existing vehicle working plans.

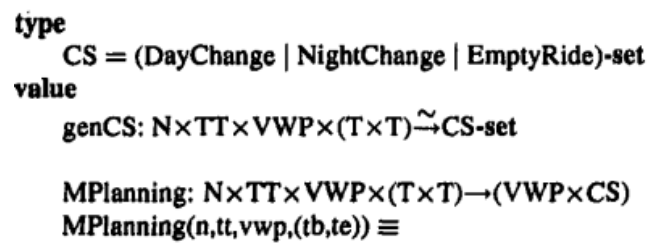

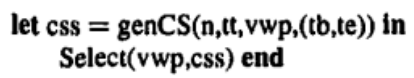

More details about this task can be found in [4].

\section{Monitoring \& Control}

Railway Net States. Lines (L) and stations (S) are composed from "smallest" rail parts called units (U) (linear, points, cross-overs, switchable crossovers). A unit define a number of connectors (linear:2, point:3, cross-overs:4). A subset of pairs of distinct connectors of a unit define paths though the unit. By a unit state $(\Sigma)$ we understand any such subset. A unit may change state. The state space of a unit is called $\Psi$. A unit is said to be closed, if it is in path state $\left(\sigma_{\{\}}\right)$of no paths.

A route (R) is a sequence of units. A route is open, if all units are in nonclosed states and if the units state paths connect [1].

By traffic (TF) we mean a function from time to net and train states (TI). A train state contains information about train position on the net, its actual and planned velocity and acceleration, etc. Our primed definition of traffic (TF') defines values that do not respect laws-of-nature (there are no "ghost trains", trains do not"jump" all over the net, etc.). Also desired railways properties (RR) are not respected in TF' (train movements only on open routes, only 
one train on a open route, obeying interlocking rules and regulations, etc.). See [3].
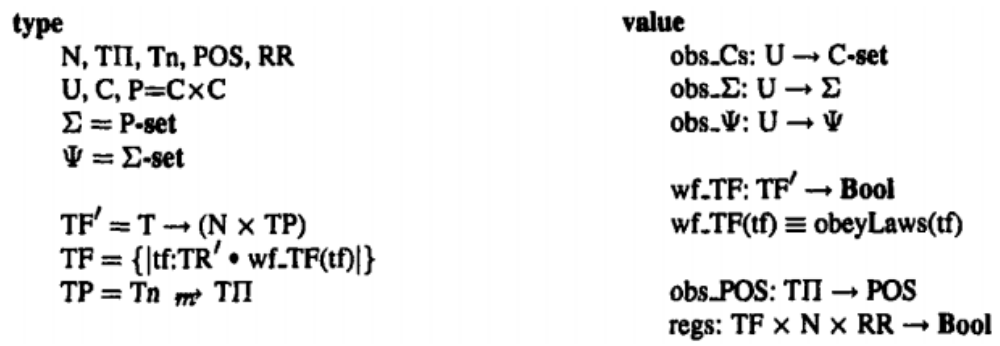

\section{From Timetable to Traffic}

The syntactic quantity a timetable (TT) denotes the semantic quantity a set of possible traffics (TF) which satisfy the timetable. See [2].

type

TR, TT, N

value

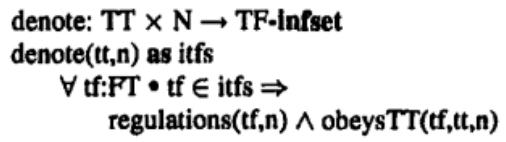

\section{From Traffic to Station Interlocking}

The above model of dynamics of units did not show how units change states. This is what we will now consider. We will use Petri Nets to model conditions for state changes of a unit.

Interlocking has to do with setting up proper routes from station approached signal to a track (platform) in the station and from these to the lines. We shall focus on one way of constructing models for proper interlocking control scheme using Place Transmission Petri Nets. Petri Net for stations can be built up from four subparts: Petri Net for units, for switches (ie., point or switchable crossover), for signals (Fig. 1), and finally Petri Net for routes. The Petri Net of a route (Fig. 2) is then a composition of all its unit, switch and signal Petri Nets - where the composition is specified by an interlocking table. Since we do not show a specific station nor its interlocking table we refrain from showing the full Petri Net [3]. The table expresses for each interesting route the state requirements for switches (points and switchable crossovers) and the requirements for signal states.

So, on one hand we have our RSL model for nets and trains states; on the other hand we have shown Petri Nets that control state spaces. In other words we have brought two different specification techniques. Hence from RLS specification of a station one can build a interlocking table and then the Petri Net. 
(a)

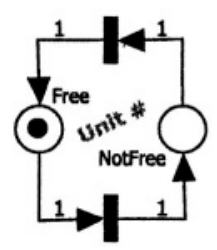

(b)

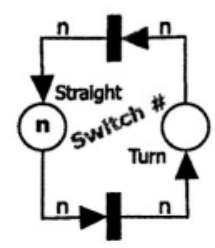

(c)

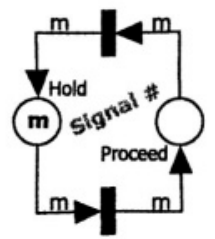

(d)

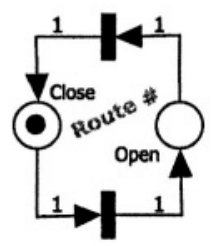

Figure 1. Petri Net for unit, switch, signal and route
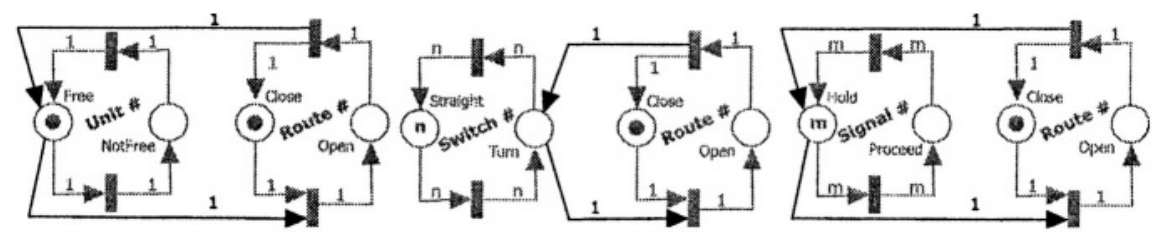

Figure 2. Adding arcs for unitlswitchisignal and route

\section{From Traffic to Line Direction Agreement}

The above RSL model of traffic did not show how certain rules could be obeyed. In this section we with to show one of the important safety properties of a railway line: that two trains are not allowed to move in opposite directions on a line. One way of ensuring this is by a so called Line Direction Agreement System (LDAS).

The externally visible behavior of the LDAS can be illustrated using Live Sequence Charts. The three entities are: Station A (SA), the Line Direction Agreement System (LDAS), and Station B (SB). The charts in Figure 3 illustrate only the partial communication as seen from Station A. The mutual exclusive control of the LDAS can illustrated by a Statechart. For more details we refer to [3]. Transitions of the Statechart corresponds to messages of the LDAS.

\section{From Traffic to Automatic Line Signalling}

Again the above RSL model of traffic did not

(a)

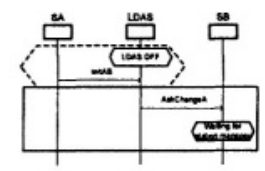

(b)

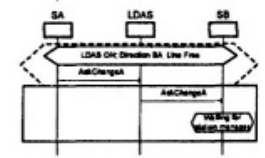

(c)

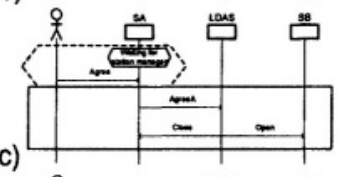

(d)

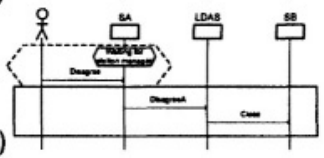

Figure 3. (a) Initial LDAS, (b) Request Direction Reversal, (c) Request Approval, (d) Request Rejection show how trains on a line can be separated. Lines connect exactly two stations and can be divided into several segments (see Fig. 4). Each segment can be either in 'Free' state (when no train is detected in the segment) or in 'Occupied' state. For each segment 
there are two signals (one in each direction of travel). With each signal we associate four possible states ('Hold', 'NextHold', 'Proceed' and 'Off').

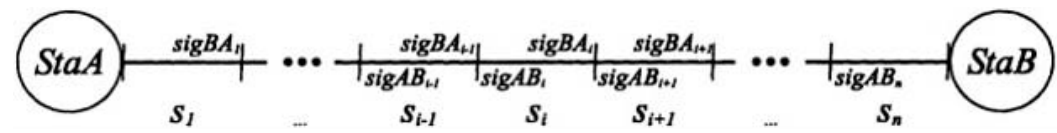

Figure 4. Signal positions on a line with $\mathrm{n}$ segments

type

TR, N, L, S, SEG, SIG

SegSt $==$ Free $\mid$ Occupied

SigSt $==$ Hold $\mid$ NextHold $\mid$ Proceed $\mid$ Off
obs_Ss: $\mathrm{L} \rightarrow$ S-set

obs_Segs: L $\rightarrow$ SEG*

obs_Sigs: SEG $\rightarrow$ SIG $\times$ SIG

obs_SegState: TR $\times$ SEG $\rightarrow$ SegSt

obs_SigState: TR $\times$ SIG $\rightarrow$ SigSt

value

Statecharts can be used to specify requirements for pair of signals along a line including pairs including station interlocking.
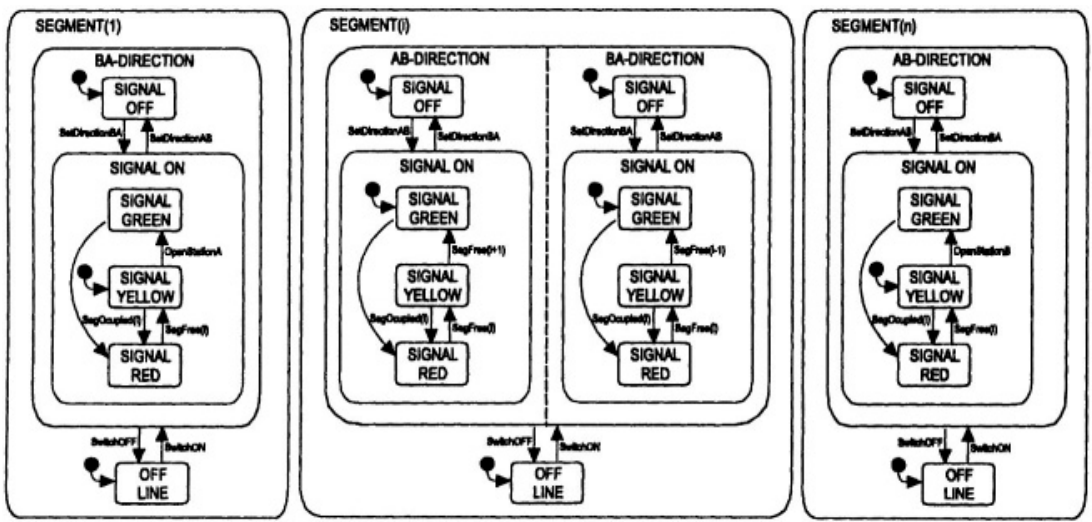

Figure 5. Sub-Statechart for Automatic signalling in lines

The Statechart for a line with three and more segments is composed from the three subparts shown on Fig. 5.

\section{Conclusion}

We have covered a part of the railway domain by rough sketching the synopses of several examples concerning scheduling \& allocation and monitoring $\&$ control aspects of railways. Our focus has been the underlying formal models. Several different formal methods, f.e. RAISE, Petri Net, StateChart, Live Sequence Chart have been used. 


\section{References}

[1] Dines Bjorner. Dynamics of Railway Nets: On an Interface between Automatic Control and Software Engineering. In CTS2003:10th IFAC Symposium on Control in Transportation Systems, Oxford, UK, August 4-6 2003. Elsevier Science Ltd. Symposium held at Tokyo, Japan. Editors: S. Tsugawa and M. Aoki.

[2] Dines Bjfirner, Jakob Braad, and Karin S. Mogensen (Eds.). Models of Railway Systems: Domain. In Thierry Lecomte and Peter Gorm Larsen, editors, FME Rail Workshop \# 5, volume \# 5 of FME Rail Workshop; Toulouse, France. FME: Formal Methods Europe, Steria, France, September 22-24 1999. (60 pages) Dept. of IT, Techn. Univ. of Denmark.

[3] Dines Bjorner, Chris George, Anne E. Haxthausen, Christian Krog Madsen, Steffen Holmslykke, and Martin Penicka. "UML"-ising Formal Techniques. In INT 2004: Third International Workshop on Integration of Specification Techniquesfor Applications in Engineering. Institut fur Softwaretechnik und Theoretische Informatik, Sekr. FR 6-1, Techn.Univ. of Berlin, Franklinstrasse 28/29, D-10587 Berlin, Germany, 28 March 2004, ETAPS, Barcelona, Spain. To be published in INT-2004 Proceedings, Springer-Verlag.

[4] Martin Penicka, Albena Kirilova Strupchanska, and Dines Bjorner. Train Maintenance Routing. In FORMS'2003: Symposium on Formal Methods for Railway Operation and Control Systems. L'Harmattan Hongrie, 15-16 May 2003. Conf. held at Techn.Univ. of Budapest, Hungary. Editors: G. Tarnai and E. Schnieder, Germany.

[5] Albena Kirilova Strupchanska, Martin Penicka, and Dines Bjorner. Railway Staff Rostering. In FORMS2003: Symposium on Formal Methodsfor Railway Operation and Control Systems. L'Harmattan Hongrie, 15-16 May 2003. Conf. held at Techn.Univ. of Budapest, Hungary. Editors: G. Tarnai and E. Schnieder, Germany. 